\title{
EPIDEMIOLOGICAL CHARACTERISTICS OF HEMOPHILIA IN THE PRE-PRIMARY PROPHYLAXIS ERA: A HISTORICAL COHORT
}

\author{
Dayenne Helena Catelli ${ }^{1}$, Júlia Plentz Portich², Adriana Celia Luz ${ }^{3}$, \\ Giovana Fecker da Costa Diaz ${ }^{3}$, Liliane Ourique de Ourique ${ }^{3}$, Daniel \\ Sander Hoffmann 4 , Guilherme Rasia Bosi ${ }^{5}$, Barbara Coiro Spessato ${ }^{1}$, \\ Leo Sekine ${ }^{6}$, Lucia Mariano da Rocha Silla ${ }^{1}$
}

\begin{abstract}
Introduction: Epidemiological studies on hemophilia in the Brazilian population are historically scarce. Despite the continuous effort made by the National Program of Inherited Bleeding Disorders to map this condition, little information is available, especially on the period prior to program conception. Therefore, the present study aims to assess the epidemiological, serological, and clinical characteristics of patients with hemophilia in the state of Rio Grande do Sul, Brazil.
\end{abstract}

Methods: A total of 455 patients had their medical records reviewed from January 1 , 2003 to December 31, 2007.

Results: We observed a remarkable prevalence of hepatitis $\mathrm{C}$ virus (HCV) infection in patients with both hemophilia $A$ and $B$, and this prevalence significantly increased along with age $(p<0.001)$. No positive anti-HCV results were observed among children younger than 5 years old. There was a significant correlation between the severity of hemophilia and the number of arthropathies in all age categories. Considering the presence of inhibitors, a significant difference was observed between age groups, as older patients had higher inhibitor titers. There was a significant correlation between mean coagulation factor consumption and the number of arthropathies in patients over 5 years old.

Conclusions: This profile analysis of patients with hemophilia reflects a gradual improvement in treatment safety and efficiency, as well as the need for continued investment in this population.

Keywords: Hemophilia; epidemiology; arthropathy; state of Rio Grande do Sul; Brazil

\section{INTRODUCTION}

Hemophilia A and B are inherited coagulation disorders caused by a quantitative deficiency of coagulation factors VIII and IX, respectively. Hemophilia is a rare disease, affecting 0.5 to 2 in 10.000 live births of boys worldwide ${ }^{1}$. Treatment costs are markedly high, especially due to the market price of coagulation factors, their considerable consumption, and the demand for specific training of health workers ${ }^{2}$. Moreover, disability resulting from inadequate treatment can be burdensome both for patients and caregivers.

The treatment of hemophilia in Brazil has evolved considerably in recent years. In the 1980s, it mainly consisted in the administration of blood components such as cryoprecipitate and fresh frozen plasma. Due to the lack of specific screening for transmissible diseases during this period, $60 \%$ of patients with hemophilia were infected by the human immunodeficiency virus (HIV) and the hepatitis $\mathrm{C}$ virus $(\mathrm{HCV})^{3}$. The acquisition and distribution of lyophilized coagulation factors started in the 1990s, but were still far from the ideal for treating the Brazilian population of patients with hemophilia. This scenario led to a whole generation of patients with serious orthopedic conditions due to hemophilic

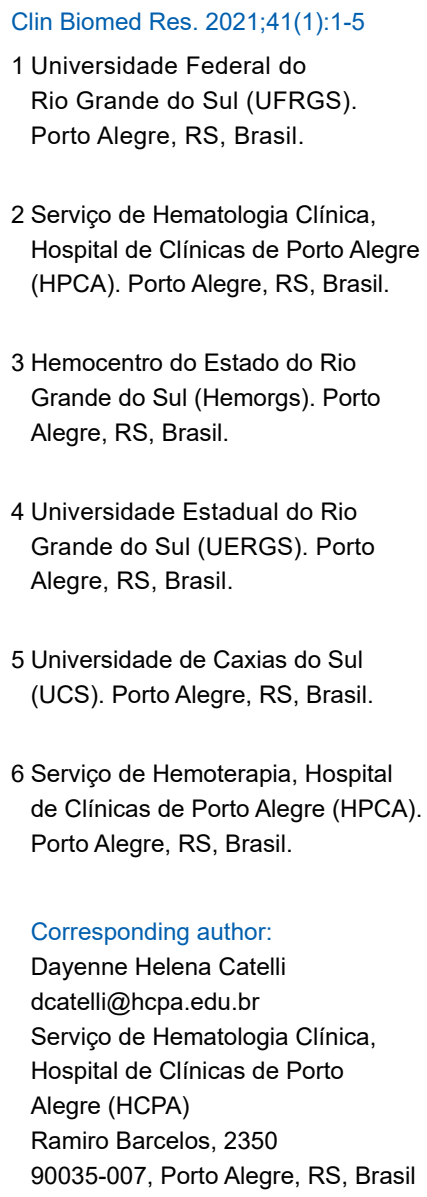

4 Universidade Estadual do Rio Grande do Sul (UERGS). Porto Alegre, RS, Brasil.

5 Universidade de Caxias do Sul (UCS). Porto Alegre, RS, Brasil.

6 Serviço de Hemoterapia, Hospital de Clínicas de Porto Alegre (HPCA). Porto Alegre, RS, Brasil.

Corresponding author: Dayenne Helena Catelli dcatelli@hcpa.edu.br Serviço de Hematologia Clínica, Hospital de Clínicas de Porto Alegre (HCPA) Ramiro Barcelos, 2350 90035-007, Porto Alegre, RS, Brasil 
arthropathy. Similarly, viral infections transmitted by blood transfusions were significantly prevalent among patients with hemophilia ${ }^{3,4}$. Beginning in the 2000s, the "Programa de Coagulopatias Hereditárias" (National Program of Inherited Bleeding Disorders) of the Brazilian Ministry of Health eventually reached the acquisition of the internationally recommended per capita amount of coagulation factor units; however, these factors were still derived from human plasma. The risk of shortages due to scarce supplies of voluntary blood donations and newly identified infection agents, such as prions, remained threats to the proper care of these patients. From 2011, the treatment of hemophilia in Brazil reached standards of excellence with the introduction of prevention programs and the acquisition of coagulation factors manufactured through recombinant technology, which were exempt from the contamination risk brought by biological agents $\mathrm{s}^{5-7}$.

The epidemiological information on patients with hemophilia in Brazil is still far from adequate ${ }^{8}$. Population-based registries play an important role in monitoring and measuring the quality of health care services and the outcomes obtained with the proposed interventions. The importance of these records for the quality of care of patients with hemophilia is clearly recognized, as they allow the understanding of clinical needs and adequate resource planning. The first national registry initiative was the creation, in 2002, of the Inherited Bleeding Disorder Program database, initially based on electronic spreadsheets. Then, the "Hemovida Coagulopatias Web" software was developed in $2009^{9}$. Unfortunately, both initiatives were handicapped by insufficient data loading. Information on the disease prior to the implementation of these initiatives is even more limited.

In the attempt to recover regional epidemiological data referring to the period when databases were not systematically updated, this study assessed characteristics of patients with hemophilia managed in a public health care reference center (Hemocentro do Rio Grande do Sul - Hemorgs) in the state of Rio Grande do Sul (RS), Brazil from 2003 to 2007.

\section{METHODS}

\section{Study design and data extraction}

This was a retrospective cohort study that assessed the medical records of patients diagnosed with hemophilia from different regions of the state of Rio Grande do Sul between January 1, 2003 and December 31, 2007. The enrolled institutions were Hemorgs and the Hemostasis Laboratory of the Genetics Department at Universidade Federal do Rio Grande do Sul (UFRGS). Hemorgs is a health care reference center assigned to conduct the Inherited Bleeding Disorders Program regionally, in association with the State Foundation for Production and Research in Health (Fundação Estadual de Produção e Pesquisa em Saúde - FEPPS). Hemorgs is an exclusive distributor of blood products acquired by the Ministry of Health and provides health assistance to registered patients. The Hemostasis Laboratory of the Genetics Department at UFRGS performs all the genetic testing and diagnosis of blood disorders in association with Hemorgs.

The date of diagnosis was defined as the date of the first confirmatory test (coagulation factor VIII or IX activity). Sociodemographic information was obtained from medical records. The presence of inhibitors and infections related to transfusions or coagulation factor infusions was evaluated. Therapeutic products such as lyophilized coagulation factors (both recombinant and derived from human plasma), antifibrinolytic agents, and blood products were assessed. Disease severity was categorized as defined by the World Federation of Hemophilia: mild (clotting factor activity between $5 \%$ and $40 \%$ ), moderate (between $1 \%$ and $5 \%$ ), or severe (less than $1 \%$ of clotting factor activity).

\section{Ethical aspects}

The project was approved by the FEPPS Ethics Committee.

\section{Statistical analysis}

Categorical variables were described as absolute and relative frequencies. Continuous variables were described as means and standard deviations in case of normal distributions, and as medians and interquartile ranges if distributed asymmetrically. The chi-squared test was used to compare proportions and the Spearman's correlation coefficient was applied to verify associations between continuous variables. An alpha error $<0.05$ was considered statistically significant. Analyses were performed on SPSS version 21.0 (SPSS Inc., Chicago, IL, USA).

\section{RESULTS}

A total of 455 records were evaluated, of which 449 had available clinical data. Demographic characteristics of the patients can be seen in Table 1. Hemophilia A (HA) was more frequent than Hemophilia B (HB) (86.5\% vs 13.5\%). Regarding disease severity, the proportions of severe (HA, $n=154$; $\mathrm{HB}, \mathrm{n}=23$ ), moderate (HA, $\mathrm{n}=77 ; \mathrm{HB}, \mathrm{n}=14$ ), and mild (HA, $n=117 ; \mathrm{HB}, \mathrm{n}=17) \mathrm{HA}$ and $\mathrm{HB}$ had no significant differences $(p=822)$. 
Table 1: Demographic characteristics of patients with hemophilia

\begin{tabular}{lc}
\hline Characteristic & $\mathbf{n ~ ( \% )}$ \\
\hline Total & $449(100 \%)$ \\
Age (mean \pm SD) & $25 \pm 16.2$ \\
Sex & \\
Male & $442(98.4 \%)$ \\
Female & $7(1.6 \%)$ \\
Ethnicity & $396(93 \%)$ \\
White & $19(4.5 \%)$ \\
Mixed ethnicity & $8(1.9 \%)$ \\
Black & \\
Hemophilia type & $384(86.5 \%)$ \\
A & $60(13.5 \%)$ \\
B & $198(85.3 \%)$ \\
\hline
\end{tabular}

Legend: SD: standard deviation.

Serological screening for infectious diseases was performed in more than $90 \%$ of the patients, and the main transfusion-transmissible viruses were investigated: HIV, HCV, human T-cell leukemia virus types 1 and 2 (HTLV 1 and 2), and hepatitis $B$ virus (measured through the hepatitis $B$ surface antigen [HBsAg] and the hepatitis $B$ core antibody [Anti-HBC]). Seropositivity prevalence can be seen in Table 2. For comparison purposes, patients were divided according to age into the following groups: less than 5 years old (yo) (HA, $n=29 ; \mathrm{HB}, \mathrm{n}=2), 5-12$ yo $(\mathrm{HA}, \mathrm{n}=63$; $\mathrm{HB}, \mathrm{n}=16), 12-18$ yo $(\mathrm{HA}, \mathrm{n}=79$; $\mathrm{HB}, \mathrm{n}=12)$, and more than 18 yo $(\mathrm{HA}, \mathrm{n}=203 ; \mathrm{HB}$, $n=30$ ). The prevalence of anti-HCV seropositivity was statistically different between age strata, being higher $(77.8 \%)$ in those over 18 yo $(p<0.001$; Pearson's chi-squared test). No positive anti-HCV case was observed among children younger than 5 yo.

Table 2: Prevalence of viral infections

\begin{tabular}{lcc}
\hline Markers & $\begin{array}{c}\text { Number of positive } \\
\text { tests (\%) }\end{array}$ & $\begin{array}{c}\text { Total number of } \\
\text { tests }\end{array}$ \\
\hline HTLV 1/2 & $8(1.9)$ & 419 \\
HBSAg & $11(2.6)$ & 419 \\
HIV & $32(7.7)$ & 418 \\
Anti-HBC & $104(24.9)$ & 418 \\
Anti-HCV & $197(47.0)$ & 419 \\
\hline
\end{tabular}

Legend: HTLV: human T-cell leukemia virus types 1 and $2 ; \mathrm{HBsAg}$ : hepatitis $B$ virus surface antigen; HIV: human deficiency virus; Anti-HBC: hepatitis B virus core antibody; Anti-HCV: hepatitis C virus antibody.
Considering arthropathies, $197(52.3 \%)$ patients with $\mathrm{HA}$ and 26 (45.6\%) patients with HB presented at least one affected joint $(p=.35$; Pearson's chi-squared test). There was a significant positive correlation between disease severity and the number of arthropathies (Rho 0.197, $p<.001$; Spearman's correlation test).

The presence of inhibitors was also evaluated. Considering patients with HA, $282(86.5 \%)$ had negative titers, $14(4.3 \%)$ had titers between 0.1 and $0.9 \mathrm{BU}$ (Bethesda unit), $28(8.6 \%)$ presented titers between 1 and $5 \mathrm{BU}$, and $2(0.6 \%)$ had titers of more than $5 \mathrm{BU}$. As for patients with HB, $55(93.2 \%)$ had negative titers, $2(3.4 \%)$ had titers between 0.1 and $0.9 \mathrm{BU}, 2(3.4 \%)$ presented titers between 1 and $5 \mathrm{BU}$ and none $(0 \%)$ had titers of more than $5 \mathrm{BU}$. There were no differences in proportion between these subgroups ( $p=.484$; Pearson's chi-squared test). When comparing titer values between age groups (Table 3 ), older patients showed significantly higher inhibitor titers $(p=0.040)$.

Table 3: Inhibitor titers by age group

\begin{tabular}{lccccc}
\hline Age (years) & $<\mathbf{5}$ & $\mathbf{5 - 1 2}$ & $\mathbf{1 2 - 1 8}$ & $\mathbf{> 1 8}$ & p value \\
\hline Inhibitor titer & 26 & 72 & 72 & 182 & \\
Negative & $(89.7)$ & $(94.7)$ & $(96.0)$ & $(87.9)$ & \\
& 2 & 4 & 2 & 25 & \\
$1-5$ & $(6.9)$ & $(5.3)$ & $(2.7)$ & $(12.1)$ & \\
& 1 & 0 & 25 & 0 & \\
& $(3.4)$ & & $(12.1)$ & & \\
$\mathrm{n}$ & 29 & 76 & 99 & 207 & 0.04 \\
\hline
\end{tabular}

The median coagulation factor dose used in the evaluated patients during the study period was 19,350 international units (IUs) (interquartile range $7,875-44,700)$. We observed a significant correlation between mean factor consumption and the number of arthropathies in the following age groups: 5-12 yo $(\mathrm{Rho}=.36, \mathrm{p}=.002) ; 12-18$ yo $(\mathrm{Rho}=.48, \mathrm{p}<001)$, and $>18$ yo $($ Rho $=.29, p<.001)$.

\section{DISCUSSION}

This study evaluated clinical and epidemiological characteristics of patients with hemophilia in the state of Rio Grande do Sul, Brazil, in a period marked by the scarcity of information regarding their profiles.

We observed an impressively high prevalence of $\mathrm{HCV}$ infections that increased across age groups. These findings revealed that, before the use of lyophilized coagulation products, therapeutic safety was an important issue. After the implementation of the use of these products and for the last 25 years, no seroconversion for HIV or HCV has been reported ${ }^{10,11}$. 
The seropositivity presented by patients in the 5-18 yo age group is likely to be due to exposure to contaminated blood products. The absence of HCV infections in children younger than 5 yo is an undeniable result of improvements in the treatment of hemophilia. Methods of purification and viral inactivation associated with a more effective screening of donors for possible viral infections through nucleic acid testing increased the safety level of blood products.

The total prevalence of $\mathrm{HCV}$ infections $(47 \%$ in patients with $\mathrm{HA}$ and $24.2 \%$ in those with $\mathrm{HB}$ ) is remarkable. Although reports from $2009-2010^{6}$ described a prevalence of $5 \%, 85 \%$ of the patients had not been not tested or did not have HCV test results on their medical records. Similarly, a $2011-2012^{5}$ report also revealed a $5 \%$ HCV seropositivity, despite describing $85 \%$ of untested patients. In the present cohort, $92 \%$ of the evaluated patients had information available regarding HCV tests. Therefore, the prevalence of $\mathrm{HCV}$ infections, albeit impressive, is more likely to represent the real-life status of this population.

Data regarding HIV infections are also conflicting. A report from 2007 showed a prevalence of $4.5 \%$ for patients with $\mathrm{HA}$ and $3.5 \%$ for those with $\mathrm{HB}$, even though only $30 \%$ of the patients had been tested ${ }^{7}$. Another report settled a prevalence of $1 \%$ for patients with both $\mathrm{HA}$ and $\mathrm{HB}$, but $80 \%$ of their patients lacked screening. The World Federation of Hemophilia estimates that around $10 \%$ of the population with hemophilia is infected with $\mathrm{HIV}^{12,13}$. This value is much closer to what was observed in our cohort, (7.7\% prevalence). Previous reports are likely a result of underreporting and testing scarcity.

Musculoskeletal bleeding is the main clinical manifestation and cause of morbidity in patients with hemophilia. If left untreated, bleeding leads to decreased range of motion and joint deformity, hampering mobility and culminating in a sedentary lifestyle and its consequences. We found at least one arthropathy in $52.3 \%$ of patients with $\mathrm{HA}$ and in $45.6 \%$ of those with $\mathrm{HB}$, without a significant difference in frequency. On the other hand, the literature indicates a 3-fold higher risk of arthropathy in patients with $\mathrm{HA}^{14}$. Two other studies with adolescents and young adults evaluated the number of arthropathies, annual average consumption of coagulation factors, frequency of bleeding, and radiological findings; their results also showed a higher severity of bleeding in patients with $\mathrm{HA}^{15,16}$. Notwithstanding, a recent study conducted with children with severe and moderate $\mathrm{HA}$ and $\mathrm{HB}$ found no differences regarding age of first exposure to coagulation factor, age of first general bleeding, and age of first articular bleeding ${ }^{17}$. This discrepancy might be due to the divergent severity criteria and methodologies adopted by these studies.

In addition, we found an intuitive relationship between increases in disease severity and the number of arthropathies, along with a direct association between mean factor consumption and the frequency of arthropathies. These results suggest that the severity of hemophilia is closely related to a residual activity of the coagulation factor.

The development of inhibitors is a widely feared complication in the treatment of hemophilia and correlates with a worse prognosis. There is still concern regarding an increased risk of inhibitors associated with the early exposure to coagulation factors performed in primary prophylaxis, as well as with the use of recombinant products when compared to plasma-derived. Testing for inhibitors is fundamental when monitoring patients with hemophilia. In fact, $85 \%$ of our population was tested and most had no detectable inhibitors ( $86.5 \%$ for $\mathrm{HA}$ and $96 \%$ for $\mathrm{HB}$ ). The testing rate and prevalence of inhibitors were higher in our study when compared to those reported by the Profile of Hereditary Coagulopathies in Brazil published in 2007 by the state of Rio Grande do $\mathrm{Sul}^{7}$. In this profile, which compiles data from 2005 and 2006, only 7 individuals with HA were tested for inhibitors, corresponding to $1.8 \%$ of the 384 -patient population; all had negative inhibitor results. On the following profile, referring to 2011-2012, these rates were improved: $72 \%$ of patients with HA were tested, with $5.95 \%$ of positive results regarding all patients. Among patients with $\mathrm{HB}, 51$ had been tested and the proportion of patients presenting inhibitors was $2.38 \%{ }^{5}$. The detection of inhibitors impacts treatment and increases its cost. When comparing our results to the reports of subsequent years ${ }^{5-7}$, we might assume that the reduction in inhibitor detection rates was mainly due to underreporting, since there was an increase in investment in programs to promote health in this population.

In the 2000s, the National Program of Inherited Bleeding Disorders of the Brazilian Ministry of Health reached the acquisition of the internationally recommended number of coagulation factor units per capita. According to the 2011 profile, the average consumption of factors VIII and IX was, respectively, 31,069 IUs and 32,105 IUs per patient. In 2012, there were marked increases of $21.1 \%$ and $15.4 \%$ in the mean consumption of factors VII and IX per patient, respectively. For the same level of correction, the number of IUs required for treating $\mathrm{HB}$ is twice that required for treating $\mathrm{HA}$; therefore, the proportion of coagulation factor consumption by patients with $\mathrm{HB}$ is clearly inferior. These findings also correlate with those reported by other studies ${ }^{18}$.

Some limitations of this study should not be overseen. As a retrospective study with data collected from medical records, missing information is an inherent bias. Indeed, the National Program of Inherited Bleeding Disorders in Brazil had an important percentage of missing data and underreporting ${ }^{5-7}$. 
Our results delineate the improvements in hemophilia treatment before and at the moment of program implementation. Unfortunately, it is reasonable to assume that underreporting has increased along the years, which renders the evaluation of the true impact of the initiative (and of the areas in need for improvement) a great challenge. It is important to reinforce that these data were obtained in the preprophylaxis era, and an ongoing study aims to verify the impact of this intervention. The awareness of health care workers on the importance of reporting hemophilia cases and treatment outcomes is crucial for extracting reliable information that underpins decision making on hemophilia in Brazil.

\section{REFERENCES}

1. Blanchette VS, Key NS, Ljung LR, Manco-Johnson MJ, van den Berg $\mathrm{HM}$, Srivastava A et al. Definitions in hemophilia: communication from the SSC of the ISTH. J Thromb Haemost. 2014;12(11):1935-9.

2. De Kleijn $P$, Odent $T$, Berntorp $E$, Hilliard P, Pasta G, Srivastava A et al. Differences between developed and developing countries in paediatric care in haemophilia. Haemophilia. 2012;18(suppl.4):94-100.

3. Ferreira AA, Leite ICG, BustamanteTeixeira MT, Guerra MR. Hemophilia A in Brazil - epidemiology and treatment developments. J Blood Med. 2014;5:175-84.

4. Antunes SV. Haemophilia in the developing world: the Brazilian experience. Haemophilia. 2002;8(3):199-204.

5. Ministério da Saúde (BR). Secretaria de Atenção à Saúde. Departamento de Atenção Hospitalar e de Urgência. Perfil das coagulopatias hereditárias no Brasil 2011-2012. Brasília (DF): Ministério da Saúde; 2014.

6. Ministério da Saúde (BR). Secretaria de Atenção à Saúde. Departamento de Atenção Especializada. Perfil das coagulopatias hereditárias no Brasil 2009-2010. Brasília (DF): Ministério da Saúde; 2012.
7. Ministério da Saúde (BR). Secretaria de Atenção à Saúde. Departamento de Atenção Especializada. Perfil das coagulopatias hereditárias no Brasil 2007. Brasília (DF): Ministério da Saúde; 2008.

8. Rezende SM, Pinheiro K, Caram C, Genovez G, Barca D. Registry of inherited coagulopathies in Brazil: first report. Haemophilia. 2009;15(1):142-9.

9. Barca DAAV, Rezende SM, Simões BJ, Pinheiro KN, Daisson T, Sternick $\mathrm{G}$ et al. Hemovida Web Coagulopatias: um relato do seu processo de desenvolvimento e implantação. Cad Saude Colet. 2010;18(3):434-5.

10. Tabor E. The epidemiology of virus transmission by plasma derivatives: clinical studies verifying the lack of transmission oh hepatitis $B$ and $C$ viruses and HIV type 1. Transfusion. 1999;39(11-12):1160-8.

11. Manucci PM. Back to the future: a recent history of haemophilia treatment. Haemophilia. 2008;14(suppl.3):10-8.

12. World Federation of Hemophilia. World Federation of Hemophilia report on the annual global survey 2012. Montreal: 2013.

13. World Federation of Hemophilia. World Federation of Hemophilia report on the annual global survey 2013. Montreal: 2014.
14. Tagariello G, lorio A, Santagostino E, Morfini M, Ruggero B, Massimo I et al. Comparison of the rates of joint arthroplasty in patients with severe factor VIII and IX deficiency: an index of different clinical severity of the 2 coagulation disorders. Blood. 2009;114(4):779-84.

15. Schulman S, Eelde A, Holmström M, Stählberg G, Odeberg J, Blombäck $M$. Validation of a composite score for clinical severity of haemophilia. J Thromb Haemost. 2008;6(7):1113-21.

16. Santagostino E, Mancuso ME, Tripodi A, Chantarangkul V, Clerici M, Garagiola I et al. Severe haemophilia with mild bleeding phenotype: molecular characterization and global coagulation profile. J Thromb Haemost. 2010;8(4):737-43.

17. Clausen N, Petrini P, ClaeyssensDonadel S, Gouw SC, Liesner R, PedNet and Research of Determinants of Inhibitor development (RODIN) Study Group. Similar bleeding phenotype in young children with haemophilia A or B: a cohort study. Haemophilia. 2014;20(6):747-55.

18. Nagel K, Walker I, Decker K, Chan AKC, Pai MK. Comparing bleed frequency and factor concentrate use between haemophilia a and B patients. Haemophilia. 2011;17(6):872-4.

Received: Sep. 29, 2020 Accepted: Nov. 6, 2020 